\title{
DETERMINATION OF CONTINUUM FRACTURE MECHANICS PARAMETERS FRO MOLECULAR DYNAMICS SIMULATIONS
}

\author{
LARISA V. STEPANOVA ${ }^{1}$, OKSANA N. BELOVA ${ }^{1}$ AND SERGEY A. BRONNIKOV ${ }^{1}$ \\ ${ }^{1}$ Samara National Research University \\ 34, Moskovskoe Shosse, Samara, Russia \\ Stepanova.1v@ssau.ru
}

Key words: Continuum Fracture Mechanics Parameters, Stress Intensity Factor, T-stress, Higher-order Coefficients, Molecular Dynamics Simulation, Over-deterministic Method.

\begin{abstract}
This study is aimed at determination of continuum fracture mechanics parameters such as stress intensity factors, T-stress and higher-order terms, from molecular dynamics simulation performed for copper plate with narrow diamond-like crack. For this purpose, an embedded atom potential (EAM) available in LAMMPS (Large-scale Atomic/Molecular Massively Parallel Simulator) molecular dynamics (MD) software is utilized to accurately describe mixed-mode crack growth. The stresses calculated from MD method using the virial theorem are used for over-deterministic method for extracting stress intensity factors, T-stress and higher - order terms of Williams series expansion. It is shown that the algorithm of the over-deterministic method can be generalized for MD calculations and gives the reasonable value of continuum fracture mechanics parameters. The obtained value of stress intensity factors, T-stresses and higher order terms are compared with the theoretical values for an infinite plate with the central crack under Mode I loading and Mixed Mode loading conditions for several values of the mixity parameter which defines the type of Mixed Mode loading.
\end{abstract}

\section{INTRODUCTION}

It is well-known that stress fields in the vicinity of the crack tip in an isotropic linear elastic material are characterized by stress intensity factors for Mode I and Mode II loadings respectively. Nowadays identification of stress intensity factors as functions of crack geometry and loading conditions is an active area of research [1-6]. Together with stress intensity factors, T-stress and higher-order terms play significant role in quantifying material failure under load. Many authors [1-6] show an essential consideration of higher order terms in Williams series expansion. Several approaches for determination of these fracture mechanics parameters have been proposed. Among them the most widely used is the over-deterministic technique which allows us to extract fracture mechanics parameters from experimental data and numerical analysis. The over-deterministic method has shown its effectiveness. Amid numerical methods molecular dynamics (MD) simulation is widely considered the best technique for modelling of atomic scale fracture [6-10]. This method is applied for atomic scale analogue to the continuum fracture mechanics parameter, namely, stress intensity factors, T-stress and higher order terms. MD simulations of fracture processes have improved the understanding of the mechanical 
origins and crack growth phenomena and have been used for computer modelling fracture processes in a variety of materials [6-10]. MD technique is used for determination fracture mechanics parameter, namely, crack propagation direction angle for Mixed Mode Loading [6]. Finally, nowadays MD method is used for determination of continuum fracture mechanics parameters [11-16]. Pioneering works on influence of the elastic T-stress in atomic modelling were the papers of Beltz and Machova [11,12] where a central crack was embedded in a rectangular atomic sample under Mode I and biaxial loading. In [13] a possible method to couple atomistic modeling of initiation of fracture in iron with displacement constraints taken from continuum considerations is discussed. In [14] it is demonstrated that measuring the Crack Tip Opening Displacement (CTOD) of single crystal silicon and iron at atomic level provides realistic estimate of the fracture toughness. In [15] fracture properties of nanoscale single-crystal silicon plates. The study []15 is based on the displacement field near the crack tip in an isotropic linear elastic body and estimates stress intensity factors from MD simulations and FE method. The authors compare results obtained by molecular dynamics and finite element methods. All critical values of stress intensity factors given by MD simulations and FE calculations based on the boundary layer model are in good agreement [15]. A novel numerical method for determination of SIFs from atomistic simulations is proposed in [16]. Using atomic coordinates to describe the displacement field about a crack tip, our method projects observed displacements onto the set of continuous displacement fields defined by the Williams expansion, with the expansion coefficients determining the SIFs. The authors of [16] show that the approach is

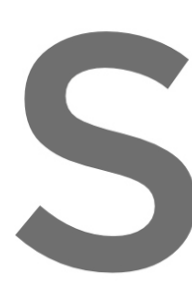
compact, has a uniq collocation and the magnitude of system have been determine discrete and heterogen parameter
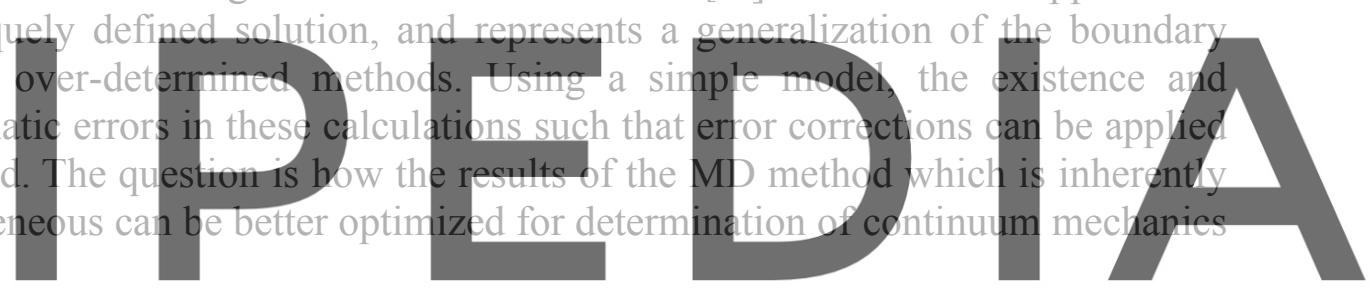

Here the numerical method for the determination of an analog to the continuum SIFs from

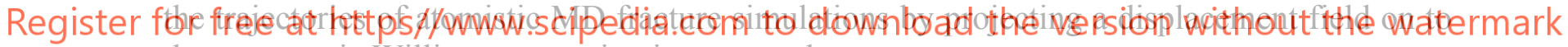
the asymptotic Williams expansion is presented.

\section{METHODOLOGY}

A series of the plate with the central crack subjected to Mixed Mode Loading were studied using the classical MD code LAMMPS. FCC Cu was selected as the model material. The EAM potential for crystalline $\mathrm{Cu}$ was applied. Copper plate with narrow diamond-like crack is modelled with periodic boundary conditions along all three axes. It is made mostly to speed up the process, because this approach showed the best performance of all. Substantial gap, bigger than energy cut-off radius was added above and below copper plate to properly simulate plain stress. It is impossible to create mathematical crack on molecular level and it is important to create shape of the crack with similar behaviour to classical mechanical crack. Diamond-like shape is applied in order to create only two stress concentrators instead of 4 in case of rectangular shape. In our work we modelled displacement of atoms in a simulation box with a scale of 1e-07 of box size every 100 timesteps. Each timestep was 0.1 ps. Higher and lower deformation rates were computed, but in nanoscale there was no difference in crack 
development and crack propagation angles. Copper plate sizes were 200, 200 and 4 unit cells in $\mathrm{x}, \mathrm{y}$ and $\mathrm{z}$ directions respectively. Additionally, distance of 50 unit cells separated copper plate from both upper and lower face of periodic box. The results of MD simulations and the stress tensor components obtained are shown in Figures 1 - 4.
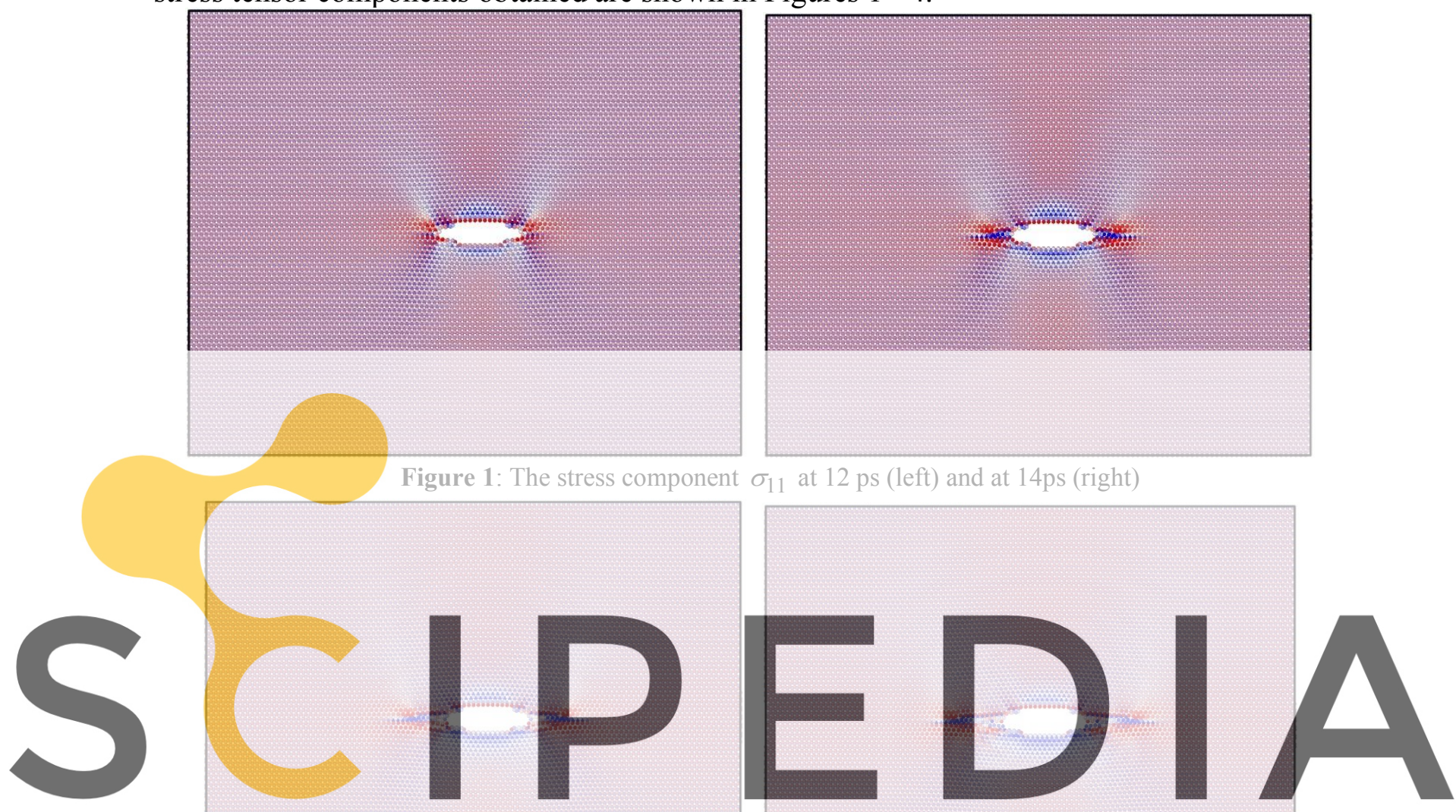

Register for free at https//www.scipedia.com to download the version without the watermark

Figure 2: The stress component $\sigma_{11}$ at $15 \mathrm{ps}$ (left) and at $16 \mathrm{ps}$ (right)
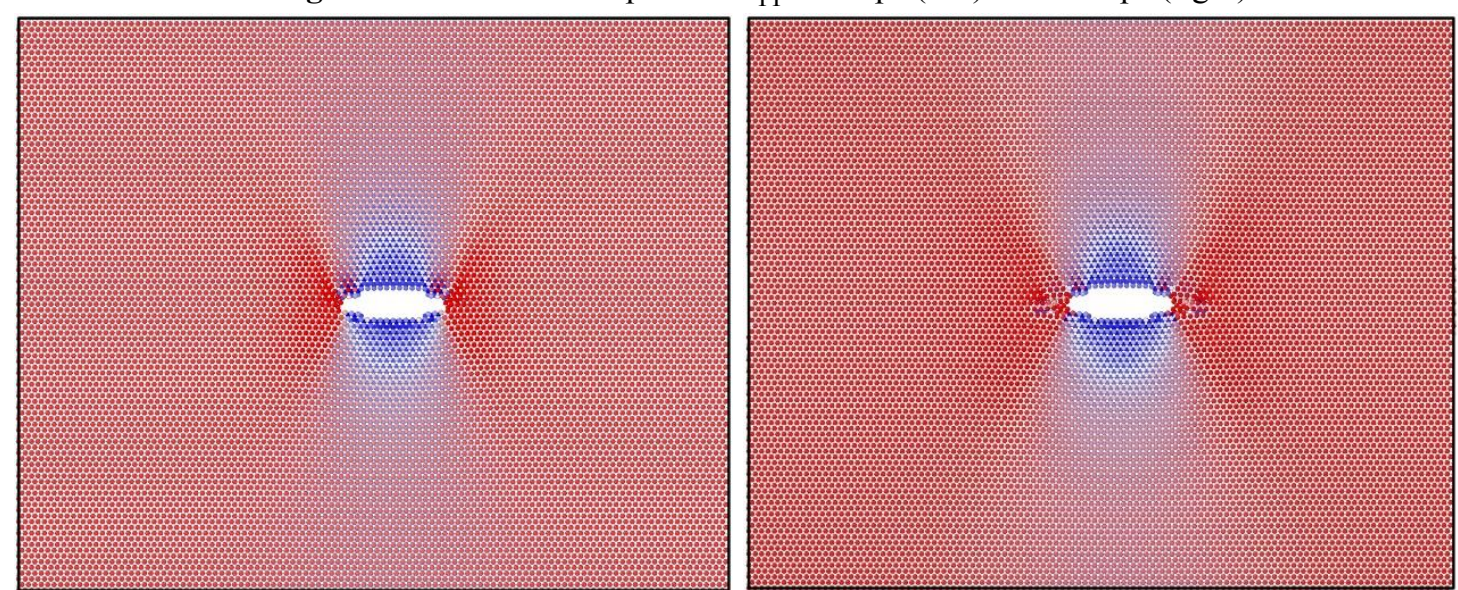

Figure 3: The stress component $\sigma_{22}$ at $12 \mathrm{ps}$ (left) and at $14 \mathrm{ps}$ (right) 

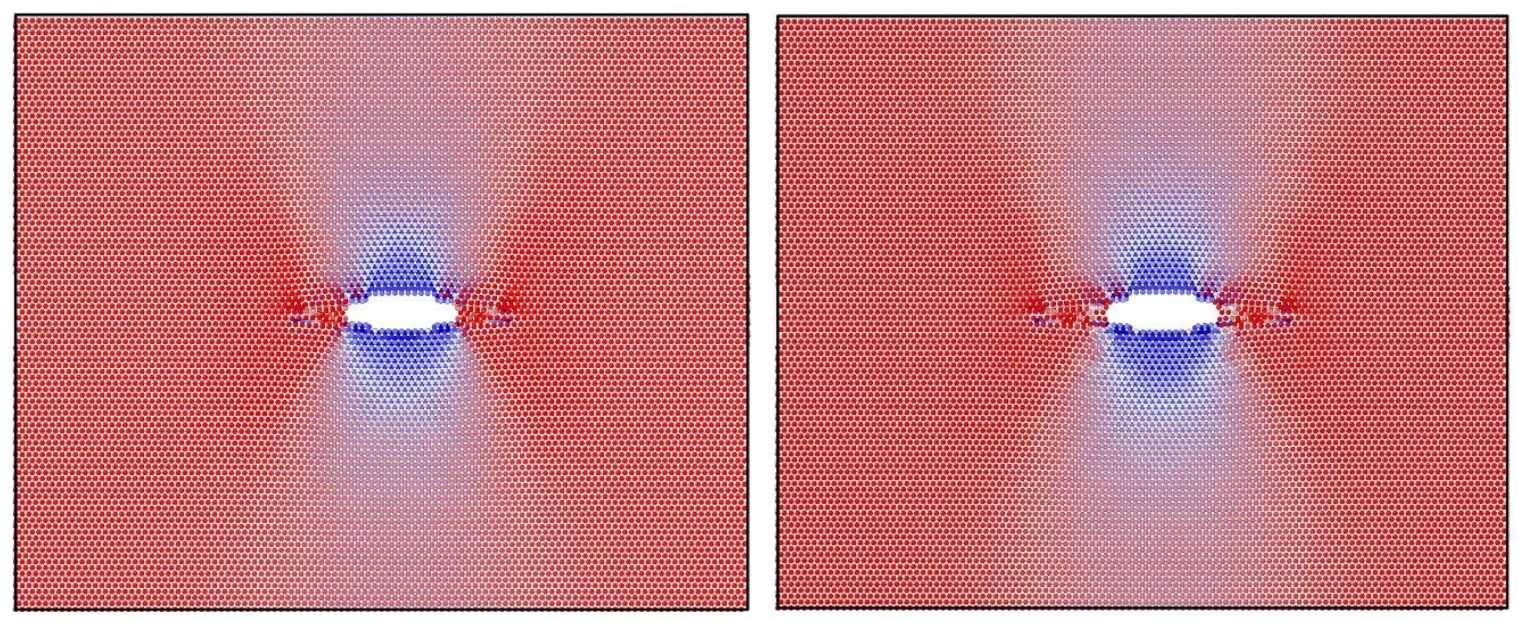

Figure 4: The stress component $\sigma_{22}$ at $15 \mathrm{ps}$ (left) and at $16 \mathrm{ps}$ (right)

\section{DETERMINATION OF FRACTURE MECHANICS PARAMETERS}

In the growing of linear fracture mechanics Williams made a principal breakthrough in the analysis of asymptotic stress field at the vicinity of the crack tip in isotropic linear elastic plane media. With the well-known eigenfunction expansion method it is possible to establish the separable variable nature of the solution and to obtain asymptotic expressions for the stress field in a plane medium with a traction-free crack submitted to mode I, mode II and mixed-

mode (mode I and mode II) loading eanditions
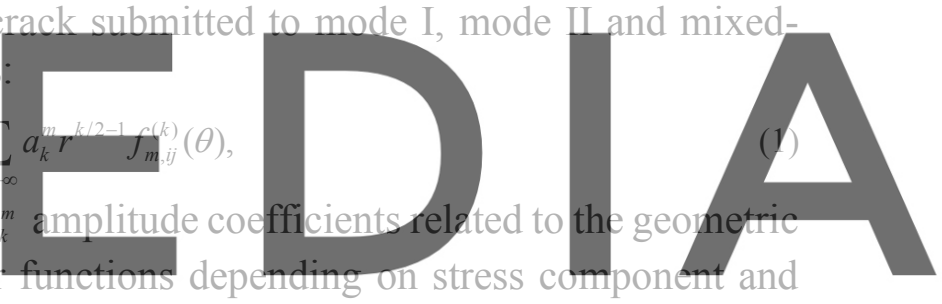

Register for free at https//WWW. scipedia.com to download the version without the watermark

$$
\begin{aligned}
f_{1,11}^{(k)}(\theta) & =\frac{k}{2}\left[\left(2+k / 2+(-1)^{k}\right) \cos (k / 2-1) \theta-(k / 2-1) \cos (k / 2-3) \theta\right], \\
f_{1,22}^{(k)}(\theta) & =\frac{k}{2}\left[\left(2-k / 2-(-1)^{k}\right) \cos (k / 2-1) \theta+(k / 2-1) \cos (k / 2-3) \theta\right], \\
f_{1,12}^{(k)}(\theta) & =\frac{k}{2}\left[-\left(k / 2+(-1)^{k}\right) \sin (k / 2-1) \theta+(k / 2-1) \sin (k / 2-3) \theta\right], \\
f_{2,11}^{(k)}(\theta) & =-\frac{k}{2}\left[\left(2+k / 2-(-1)^{k}\right) \sin (k / 2-1) \theta-(k / 2-1) \sin (k / 2-3) \theta\right], \\
f_{2,22}^{(k)}(\theta) & =-\frac{k}{2}\left[\left(2-k / 2+(-1)^{k}\right) \sin (k / 2-1) \theta+(k / 2-1) \sin (k / 2-3) \theta\right], \\
f_{2,12}^{(k)}(\theta) & =\frac{k}{2}\left[-\left(k / 2-(-1)^{k}\right) \cos (k / 2-1) \theta+(k / 2-1) \cos (k / 2-3) \theta\right] .
\end{aligned}
$$

The multi-parameter fracture mechanics concept consists in the idea that the crack-tip stress field is described by means of the Williams expansion (1). In this work central crack in an infinite plane medium is considered. To obtain the coefficients of the truncated Williams series expansion (1) the set of points belonging to concentric circles were chosen from MD simulations as shown in Figures 5 and 6. The number of points was varying from 219 to 657 
depending on the selected layer of the model and time step.

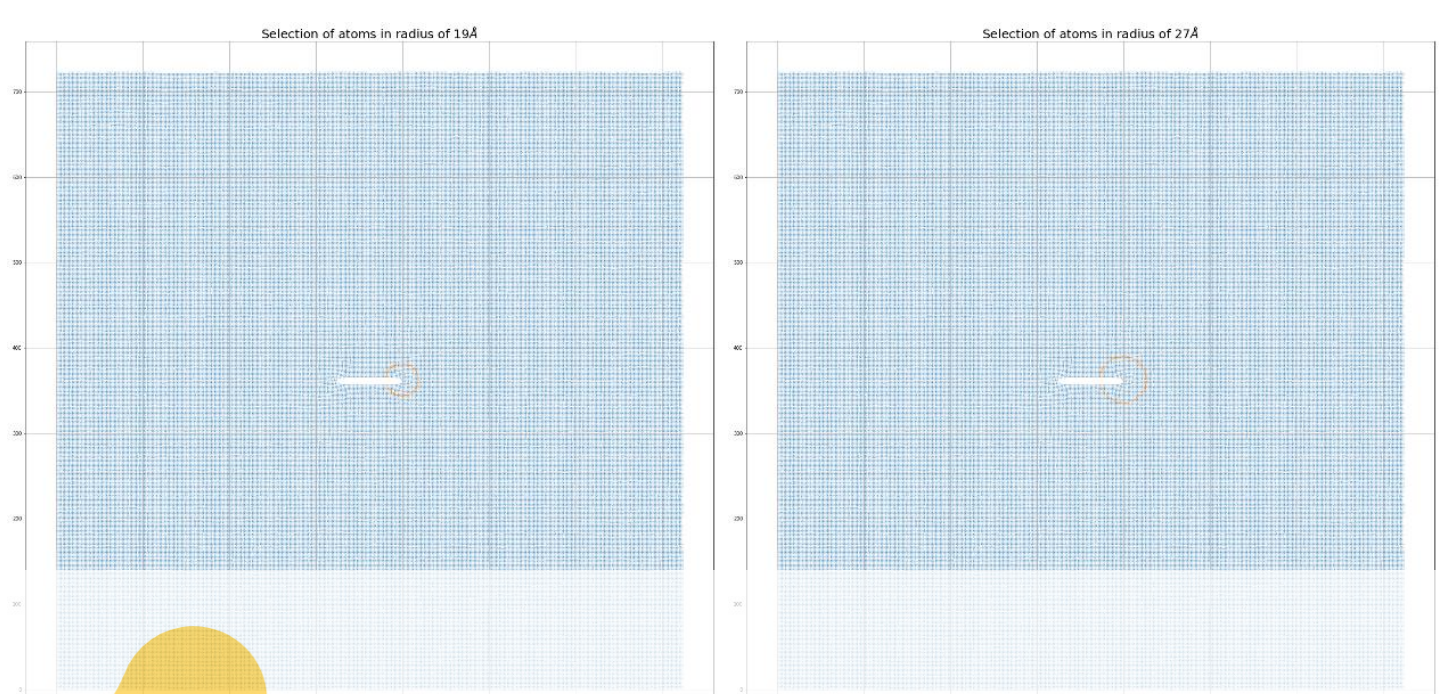

Figure 5: Selection of points taken from MD simulation at different distances from the crack tip
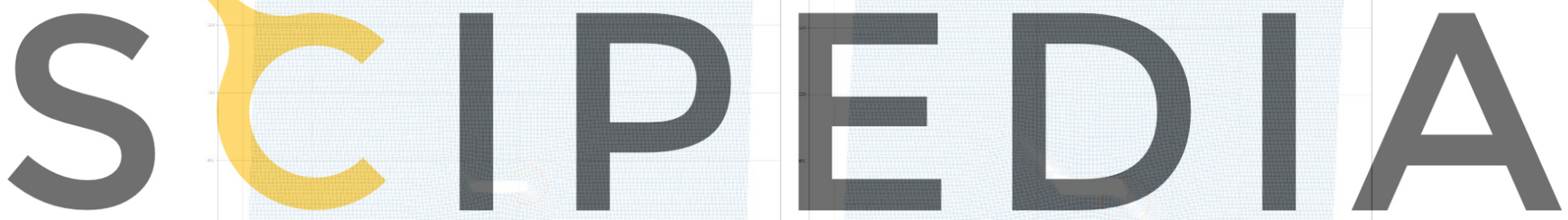

Register for free at https//www.scipedia.com to download the version without the watermark
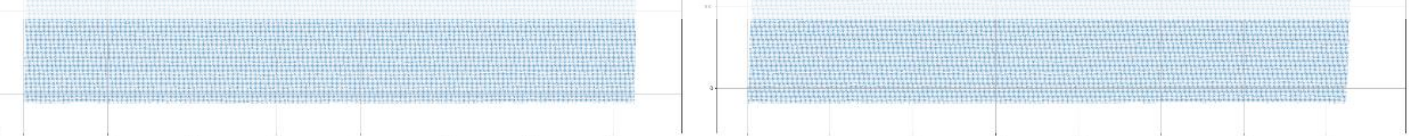

Figure 6: Selection of points taken from MD simulation at different distances from the crack tip (Mixed Mode loading)

Having obtained the set of points the procedure of the over-deterministic method can be implemented.

As it is noted in [17] the basic principle of the over-deterministic method is the use of a large number of numerical data points in order to calculate the crack tip parameters. This is done by forming an algebraic system of equations where the number of equations is more than the number of unknowns. In this case the over-deterministic system of equations is encountered. In the framework of using the over-deterministic method to determine the coefficients of (1) the values of stresses can provide the necessary set of equations. The over - deterministic technique assumes more equations than unknowns in order to obtain more accurate values. This implies that one can form an over deterministic system. Taking data from different points at different 
distances from the crack tip is allowed as higher order terms are included in the stress equations. The algorithm is implemented using in the mathematical software Maple. One can use the described approach and present equation (1) in the matrix form as

$$
\sigma=C A
$$

The closed form solution of (4) for the unknown vector of fracture mechanics parameters A can be written as

$$
A=\left(C^{T} C\right)^{-1} C^{T} \sigma
$$

where $A=\left(C^{T} C\right)^{-1} C^{T}$ is the pseudo-inverse of matrix $\mathrm{C}$. The coefficients A are estimated by minimizing the objective function which is of quadratic form for stress expression in terms of unknown parameters:

$$
J(A)=(\sigma-C A)^{T}(\sigma-C A) / 2
$$

The over-deterministic method allows us to calculate the high-order coefficients of the multipoint Williams series expansion (1) where the first five coefficients for Mode I crack have been kept. The results are $a_{1}^{1}=72.528\left(\mathrm{~Pa} / \mathrm{cm}^{3 / 2}\right), \quad a_{2}^{1}=-27.517\left(\mathrm{~Pa} / \mathrm{cm}^{2}\right), \quad a_{3}^{1}=21.406\left(\mathrm{~Pa} / \mathrm{cm}^{5 / 2}\right)$, $a_{4}^{1}=-0.033\left(\mathrm{~Pa} / \mathrm{cm}^{3}\right), a_{5}^{1}=-2.844\left(\mathrm{~Pa} / \mathrm{cm}^{7 / 2}\right)$. The values of the higher order terms for Mixed

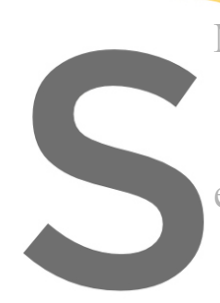

\section{Mode loading with the mixity parameter}
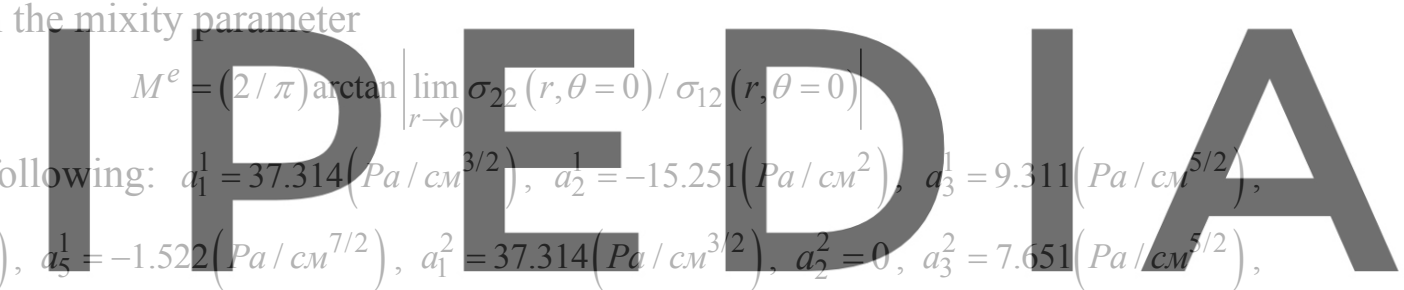

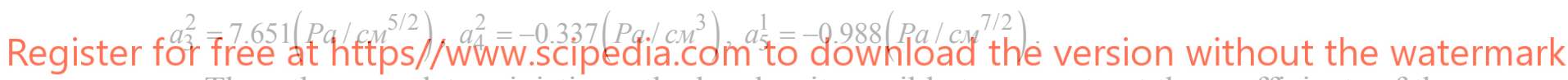

Thus, the over-deterministic method makes it possible to reconstruct the coefficients of the multi-point Williams series expansion from MD simulations.

\section{CONCLUSIONS}

The study presents the method of determination of continuum fracture mechanics parameters (stress intensity factors, T-stress and higher-order terms of Williams series expansion of the stress field in the vicinity of the crack tip in an isotropic linear elastic materials) using molecular dynamics simulations.

Based on the procedure of the over-deterministic method the continuum fracture mechanics parameters for Mode I and Mixed Mode loading are found. Reconciliation of continuum and atomistic models is demonstrated.

\section{REFERENCES}

[1] Patil, P., Vysasarayani, C.P. and Ramji, M. Linear least squares approach for evaluating crack tip fracture parameters using isochromatic and isoclinic data from digital 
photoelasticity. Optics and Lasers in Engineering (2017) 93: 182-194.

[2] Hello, G. Derivation of complete crack-tip stress expansions from Westergaard-Sanford solutions. International Journal of Solids and Structures (2018) 144-145: 265-275.

[3] Stepanova, L.V. The algorithm for the determination of the Williams asymptotic expansion coefficients for notched semidiscs using the photoelasticity method and finite element method. AIP Conference Proceedings (2020) 2216: 020013.

[4] Miarka, P., Cruces, A.S., Seitl, S., Malikova L. and Lopez-Crespo P. Evaluation of the SIF and T-stress values of the Brazilian disc with a central notch by hybrid method. International Journal of Fatigue (2020) 135: 105562.

[5] Liao, M. and Zhang, P. An improved approach for computation of stress intensity factors using the finite element method. Theoretical and Applied Fracture Mechanics (2019) 101: 185-190.

[6] Stepanova, L. and Bronnikov, S. A computational study of the mixed-mode crack behavior by molecular dynamics method and the multi-Parameter crack field description of classical fracture mechanics. Theoretical and Applied Fracture Mechanics (2020) 109: 102691

[7] Zhou, X., Yu, D. Jacobson, and Thompson, G.B. A molecular dynamics study on the stress generation during thin film growth. Applied Surface Science (2019) 469: 537 -552.

[8] Dewapriya, M.A.N. and Rajapakse, R.K.N.D. Atomistic and continuum modelling of stress field at an inhomogeneity in graphene. Materials and Design (2018) 160: 718-730.

[9] Roy, S. and Roy, A. A computational investigation of length-scale effects in the fracture

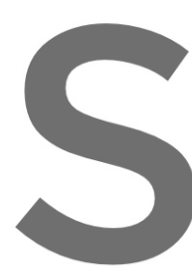
behavior of a graphene sheet using the atomistic J-integral. Engineering Fracture
Mechanics (2019) 207:165-180.
Singh, D., Sharma, P. and Parashar, A. Atomistic simulations to study crack tip
behavior in single crystal of bee niobium and hep zirconium. Current Applied Phosics
(2019) 19:37-43.
Beltz, G.E. and Machova, A. Reconciliation of continum and atomistic models for the
ductile versus brittle response of iron. Modelling and Simulation in Materials Science and

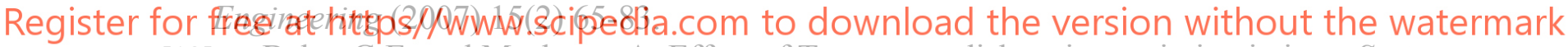

[12] Beltz, G.E. and Machova, A. Effect of T-stress on dislocation emission in iron. Scripta Mater (2004) 50(4): 483-487.

[13] Ersland, C.H., Thaulow, C., Vatne, I.R. and Ostby, E. Atomistic modeling of micromechanisms and T-stress effects in fracture of iron. Engineering Fracture Mechanics (2012) 79: 180-190.

[14] Thaulow, C. Application of CTOD in atomistic modeling of fracture. Procedia Materials Science (2014) 3: 1542-1547.

[15] Fang, Wei, Xie, H., Yin, F., Li, J., Khan, D.F. and Fang, Q. Molecular dynamics simulation of grain boundary geometry on crack propagation of bi-crystal aluminum. Materials Science \& Engineering A (2016) 666: 314-319.

[16] Wilson, M.A., Grutzik S.J. and Chandross M. Continuum stress factors from atomistic fracture simulations. Comput. Methods Appl. Mech. Engrg (2019) 354: 732-749.

[17] Aytollahi, M.R., Nejati, M and Ghouli. S. The finite element over-deterministic method to calculate the coefficients of crack tip asymptotic fields in anisotropic planes Engineering Fracture Mechanics (2020) 231: 106982. 